PROCEEDINGS OF THE

AMERICAN MATHEMATICAL SOCIETY

Volume 124, Number 10, October 1996

\title{
FIXED POINTS AND POWERS OF SELF-MAPS OF $H$-SPACES
}

\author{
GREGORY LUPTON AND JOHN OPREA
}

(Communicated by Thomas Goodwillie)

\begin{abstract}
We give a new proof of the following result due to Duan: Let $f: X \rightarrow X$ be any self-map of a finite connected $H$-space. Then $f^{k}$ has a fixed point if $k \geq 2$. Our proof is based on an approach due to Steve Halperin, whereby the Lefschetz number of a self-map is expressed in terms of the eigenvalues of the induced homomorphism of rational homotopy groups. This allows us to give a considerably shorter proof which avoids most of the technicalities of the original proof.
\end{abstract}

\section{$\S 1$. INTRODUCTION AND NOTATION}

Suppose that $(X, \mu)$ is a connected $H$-space with homotopy unit and that $f_{1}, f_{2}$ : $X \rightarrow X$ are self-maps. Their product is defined as the composition

$$
X \stackrel{\Delta}{\rightarrow} X \times X \stackrel{f_{1} \times f_{2}}{\longrightarrow} X \times X \stackrel{\mu}{\rightarrow} X .
$$

Using this product we form inductively the $k^{\text {th }}$ power of a self-map $f$, denoted $f^{k}$, by taking $f_{1}=f$ and $f_{2}=f^{k-1}$. The following result is proved in [Dua $]$ :

Theorem. Let $f: X \rightarrow X$ be a self-map of a finite, connected $H$-space. For each $k \geq 2$, the $k^{\text {th }}$ power of $f$ has a fixed point.

Recall that the Lefschetz number of a self-map $g: X \rightarrow X$, denoted $L(g)$, is defined to be $L(g)=\sum_{i=0}^{\operatorname{dim} X}(-1)^{i} \operatorname{trace}\left(g^{i}\right)$, where $g^{*}: H^{*}(X ; \mathbb{Q}) \rightarrow H^{*}(X ; \mathbb{Q})$ is the induced homomorphism on rational cohomology. Suppose that $X$ is a compact ANR. Then the Lefschetz fixed point theorem holds and says that if $L(g) \neq 0$, then $g$, and any map homotopic to $g$, has a fixed point [Bro, p.42]. We emphasize the point about maps homotopic to $g$ because we shall work with induced maps of homotopy groups and thus require basepoint preserving maps. Given any $g: X \rightarrow$ $X$, by standard methods we can replace it by a basepoint preserving $\tilde{g}: X \rightarrow X$ with $g \simeq \tilde{g}$. These maps then have the same Lefschetz number. We shall see below that $L(\tilde{g})$, and thus $L(g)$, may be calculated from the induced homomorphism $\tilde{g}_{\#}: \pi_{\#}(X) \rightarrow \pi_{\#}(X)$. In the sequel, we will blur the distinction between the homotopic maps $g$ and $\tilde{g}$ and refer to the induced homomorphism of homotopy groups $g_{\#}: \pi_{\#}(X) \rightarrow \pi_{\#}(X)$.

Duan proves that $L\left(f^{k}\right) \neq 0$ for each $k \geq 2$, by a direct analysis of the induced homomorphism of cohomology. In doing so, he encounters considerable technical

Received by the editors October 20, 1994 and, in revised form, April 3, 1995.

1991 Mathematics Subject Classification. Primary 55M20, 55P45, 55P62.

Key words and phrases. Fixed point, Lefschetz number, $H$-space, rational homotopy.

(C)1996 American Mathematical Society 
difficulty. In this paper, we use an approach based not on the induced homomorphism of cohomology, but rather on the induced homotopy homomorphism. This is made possible by a result of Steve Halperin. Actually, we prove a more general result and obtain Duan's theorem as a corollary. By applying this approach we advertise Halperin's beautiful rational homotopy theoretic formula to fixed point theorists, to whom at present it seems unknown. On the other hand, restricting to the context of Duan's result allows us to present an interesting special case of Halperin's formula that omits the spectral sequence technicalities in Halperin's proof. As a consequence of adopting the approach made possible by Halperin's formula, we can identify explicitly the 'characteristic polynomial' that appears in Duan's proof as the characteristic polynomial of the induced homotopy homomorphism (cf. also some of the results of [Dua $]$ ). That we are able to do so is the key to avoiding most of the technicalities in his proof.

\section{§2. REVieW of RATIONAL homotopy}

We first review some basics of rational homotopy theory — see [G-M] for more details. Recall that to any space $X$, with finite-type rational homology, is associated a minimal model $(\Lambda V, d)$, where $V$ is a positively graded vector space and $\Lambda V$ is a free differential graded commutative algebra (DGA) which is polynomial on even degree generators, exterior on odd degree generators and which has a decomposable differential $d$. For a nilpotent space (e.g. an $H$-space or a simply connected space), the minimal model $(\Lambda V, d)$ 'models' $X$ in the sense that there are natural isomorphisms $H^{*}(X ; \mathbb{Q}) \cong H^{*}((\Lambda V, d))$ and $\operatorname{Hom}\left(\pi_{\#}(X), \mathbb{Q}\right) \cong V$. The minimal model of $X$ is constructed from a (non-free) differential graded commutative algebra of rational polynomial forms $A^{*}(X)$, akin to de Rham forms on a smooth manifold. The construction results in a homomorphism $\rho:(\Lambda V, d) \rightarrow A^{*}(X)$ which induces an isomorphism of cohomology. This map $\rho$ enjoys the following lifting property: Given a DGA homomorphism $\phi: \mathcal{B} \rightarrow A^{*}(X)$ which induces a cohomology isomorphism, there is a lift $\tilde{\rho}:(\Lambda V, d) \rightarrow \mathcal{B}$ with $\phi \circ \tilde{\rho} \simeq \rho$. Here, ' $\simeq$ ' denotes the important notion of DGA-homotopy. This lifting property, together with the fact that a cohomology isomorphism between minimal models is in fact an isomorphism, is sufficient to establish the uniqueness of the minimal model up to isomorphism. Furthermore, a map of spaces $f: Y \rightarrow Z$ induces (in the usual fashion) a map of forms $f^{*}: A^{*}(Z) \rightarrow A^{*}(Y)$. From the lifting property again, we obtain a map of minimal models $\left(\Lambda V_{Z}, d_{Z}\right) \rightarrow\left(\Lambda V_{Y}, d_{Y}\right)$, unique up to DGA-homotopy. Therefore, in particular, we may study self-maps $g: X \rightarrow X$ on the minimal model level. Let $g: X \rightarrow X$ be a self-map and $g_{\#} \otimes 1: \pi_{\#}(X) \otimes \mathbb{Q} \rightarrow \pi_{\#}(X) \otimes \mathbb{Q}$ be the rationalized induced homomorphism on homotopy groups. The map $g$ induces an endomorphism of the minimal model, denoted $G:(\Lambda V, d) \rightarrow(\Lambda V, d)$. Now let $I(\Lambda V)=\Lambda V / \Lambda^{+} V \cdot \Lambda^{+} V$ denote the vector space of indecomposables of $\Lambda V$. If

$$
I(G): I(\Lambda V) \rightarrow I(\Lambda V)
$$

denotes the map of indecomposables induced by $G$, then $I(G)$ may be identifed with $g_{\#} \otimes 1$.

A space $X$ is elliptic if both $\pi_{\#}(X) \otimes \mathbb{Q}$ and $H^{*}(X ; \mathbb{Q})$ are finite-dimensional. In what follows, we consider elliptic spaces of a particular kind, namely those whose rational homotopy is zero in even degrees. In this case, $X$ has minimal model $(\Lambda V, d)$ with $V=0$ in even degrees. We say a graded vector space $V$ is oddly graded if it is zero in even degrees and we say a minimal model $(\Lambda V, d)$ is oddly 
generated if the vector space $V$ is oddly graded. A finite $H$-space is elliptic and has oddly generated minimal model. Furthermore, in the minimal model of an $H$-space, the differential $d$ is zero. This follows from Hopf's theorem on $H$-spaces: The cohomology algebra of a connected finite $H$-space is an exterior algebra on odd generators [Spa, p.269]. Indeed, the exterior cohomology algebra $H^{*}(X ; \mathbb{Q})$ may be mapped cohomologically isomorphically into $A^{*}(X)$ by taking generators to representing cocycles and then extending freely. The lifting theorem mentioned above then provides a map $(\Lambda V, d) \rightarrow H^{*}(X ; \mathbb{Q})$ which must also be a cohomology isomorphism and, hence, an isomorphism of DGA's.

In addition to $H$-spaces, there are many examples of elliptic spaces with oddly generated minimal model. Examples of such spaces may be constructed by taking principal $K$-bundles over $H$, where $K$ and $H$ are compact Lie groups, or any finite sequence of such bundles.

\section{§3. Eigenvalues of the Rationalized INDUCED HOMOMORPHISM ON HOMOTOPY}

Let $g: X \rightarrow X$ be any self-map of an elliptic space. Halperin's result [Hal, Th.3] describes the Lefschetz number $L(g)$ in terms of the eigenvalues of the rationalized induced homomorphism on homotopy groups $g_{\#} \otimes 1: \pi_{\#}(X) \otimes \mathbb{Q} \rightarrow \pi_{\#}(X) \otimes \mathbb{Q}$. Indeed, in the case of a space $X$ whose rational homotopy is zero in even degrees, Halperin's formula is the one we give below. In this section we make an observation about the eigenvalues of an induced homomorphism $g_{\#} \otimes 1$, in a quite general setting.

We begin with the remark that an integral matrix $A$ has no non-integral, rational eigenvalues. This follows from the 'rational root test' [Hun, Th.4.16] applied to the characteristic polynomial $\operatorname{det}(\lambda I-A)$, which is monic with integer coefficients. Now let $X$ be any space with $\pi_{i}(X)$ a finitely-generated abelian group for each $i$. For example, $X$ could be a finite $H$-space or a simply connected finite complex. Pick a (module) basis for the free part of $\pi_{\#}(X)$. These generators give a corresponding (vector space) basis for the rational homotopy groups $\pi_{\#}(X) \otimes \mathbb{Q}$. Consider a self-map $g: X \rightarrow X$, together with the following commutative diagram:

$$
\begin{array}{ccc}
\pi_{\#}(X) & \stackrel{g_{\#}}{\longrightarrow} & \pi_{\#}(X) \\
\downarrow & & \downarrow 1 \\
\pi_{\#}(X) \otimes \mathbb{Q} & \stackrel{g_{\#} \otimes 1}{\longrightarrow} & \pi_{\#}(X) \otimes \mathbb{Q} .
\end{array}
$$

With respect to our basis of $\pi_{\#}(X) \otimes \mathbb{Q}$, the linear transformation $g_{\#} \otimes 1$ has an integral matrix. From our earlier remark we conclude the following:

Lemma. For any self-map $g: X \rightarrow X$, the rationalized induced homomorphism $g_{\#} \otimes 1$ has no non-integral, rational eigenvalues.

\section{$\S 4$. The Lefschetz number And a Formula of Halperin}

We now relate the eigenvalues of $g_{\#} \otimes 1$ to the Lefschetz number of $g, L(g)$. This is done by using a special case of Halperin's formula [Hal, Th.3], which we prove by specializing his argument. Our proposition generalizes a somewhat well-known result, in which one considers an endomorphism of exterior algebras $G: \Lambda V \rightarrow \Lambda V$ that is induced by a linear map $V \rightarrow V$ (cf., for example, [Lan, p.436, Ex.7]). Here, we start with a DGA map which is not necessarily induced by an underlying linear 
map. We present the result with its proof for completeness and also to preserve the spirit of Halperin's result.

Proposition. Let $(\Lambda V, d)$ be a minimal model with $V$ an oddly graded, finite dimensional vector space. Let $G:(\Lambda V, d) \rightarrow(\Lambda V, d)$ be any DGA endomorphism and let $G^{*}$ denote the induced endomorphism of cohomology. Suppose that the induced map on the vector space of indecomposables, $I(G): I(V) \rightarrow I(V)$, has eigenvalues $\left\{\beta_{j}\right\}$. Then the Lefschetz number of $G^{*}, L\left(G^{*}\right)=\sum_{i}(-1)^{i}$ trace $\left(G^{* i}\right)$, satisfies

$$
L\left(G^{*}\right)=\prod\left(1-\beta_{j}\right) .
$$

Proof. Denote by $L(G)$ the Lefschetz number of the DGA homomorphism $G$, i.e., $L(G)=\sum_{i}(-1)^{i}$ trace $\left(G^{i}\right)$. We tensor with the complex numbers and identify $G=G \otimes 1$. Following Halperin, decompose $G$ into its semisimple and nilpotent parts, $G=G_{s}+G_{n}$. Now, for each $i, G^{i}$ and $G_{s}^{i}$ have the same trace, so we have $L(G)=L\left(G_{s}\right)$. This is the crucial step in our argument, for it allows us to restrict attention to a set of generators for $\Lambda V$. Since $G_{s}$ is semisimple, we can pick algebra generators $\left\{x_{j}\right\}$ for $\Lambda V$ which are eigenvectors of $G_{s}$. If $G_{s}\left(x_{j}\right)=\beta_{j} x_{j}$ for each $j$, then we see easily that $L\left(G_{s}\right)=\prod\left(1-\beta_{j}\right)$.

On the other hand, projecting the generators $\left\{x_{j}\right\}$ to the vector space of indecomposables gives a basis $\left\{\bar{x}_{j}\right\}$, say, with $I\left(G_{s}\right)\left(\bar{x}_{j}\right)=\beta_{j} \bar{x}_{j}$ for each $j$. In other words, the $\left\{\beta_{j}\right\}$ are also the eigenvalues of $I\left(G_{s}\right)$. So, $L(G)=L\left(G_{s}\right)=\prod\left(1-\beta_{j}\right)$, where the $\left\{\beta_{j}\right\}$ are the eigenvalues of $I\left(G_{s}\right)$.

We have shown that the right formula holds for $L(G)$ using the eigenvalues of $I\left(G_{s}\right)$. Thus, it just remains to show that the $\left\{\beta_{j}\right\}$ are also the eigenvalues of $I(G)$. This follows because $G$ is an algebra map and so preserves the subspace of decomposables. Therefore, passing to the quotient $I(\Lambda V)$ preserves semisimplicity. That is, $I(G)_{s}=I\left(G_{s}\right)$. Thus $I\left(G_{s}\right)$ is the semisimple part of $I(G)$ and, hence, the eigenvalues of $I\left(G_{s}\right)$ are also those of $I(G)$. So far, we have identified $L(G)$ and the product $\Pi\left(1-\beta_{j}\right)$. The proposition now follows from an application of the Hopf trace formula [Bro, p.6] to the 'cochain map' $G$ and its induced cohomology homomorphism $G^{*}$.

\section{§5. A proof of Duan's Result}

With the above preliminaries, we turn to the main result:

Theorem. Let $X$ be an elliptic space with oddly generated minimal model and let $g: X \rightarrow X$ be any self-map. If the induced homomorphism on rational homotopy, $g_{\#} \otimes 1: \pi_{\#}(X) \otimes \mathbb{Q} \rightarrow \pi_{\#}(X) \otimes \mathbb{Q}$, does not have 1 as an eigenvalue, then $L(g) \neq 0$ and so $g$ has a fixed point.

Proof. Suppose $X$ has oddly generated minimal model $(\Lambda V, d)$. Let $G$ denote the map of the minimal model corresponding to $g$, so that $I(G)$ may be identified with $g_{\#} \otimes 1$ and $G^{*}$ with $g^{*}$. Then by the Proposition $L(g)=\prod\left(1-\beta_{j}\right)$, and the $\beta_{j}$ may now be taken to be the eigenvalues of the rationalized induced homomorphism on homotopy groups. Thus, if $g_{\#} \otimes 1$ does not have 1 as an eigenvalue, then $L(g)$ is not zero.

Corollary. Let $f: X \rightarrow X$ be any self-map of a finite, connected $H$-space $X$. Then the $k^{\text {th }}$ power of $f, f^{k}$, has a fixed point for each $k \geq 2$. 
Proof. Denote the identity map of $X$ by $\iota$. We can write $f^{k}=\iota^{k} \circ f$. As is wellknown and easy to see, we have induced on homotopy $\left(\iota^{k}\right)_{\#}=\times k: \pi_{\#}(X) \rightarrow$ $\pi_{\#}(X)$. Hence $\left(f^{k}\right)_{\#}=k f_{\#}: \pi_{\#}(X) \rightarrow \pi_{\#}(X)$ and it follows that, if we denote the eigenvalues of $f_{\#}$ by $\left\{\beta_{j}\right\}$, then the eigenvalues of $\left(f^{k}\right)_{\#} \otimes 1$ are $\left\{k \beta_{j}\right\}$. The only way such an eigenvalue $k \beta_{j}$ can equal 1 is if $\beta_{j}=\frac{1}{k}$. By the Lemma, however, $f_{\#} \otimes 1$ has no non-integral, rational eigenvalues. Therefore, for $k \geq 2,\left(f^{k}\right)_{\#} \otimes 1$ does not have 1 as an eigenvalue and the result follows.

\section{ACKNOWLEDGMENTS}

Thanks to Martin Arkowitz for pointing out Duan's result to us and also to Robert Brown for discussions concerning the result. The referee made numerous helpful comments which have improved the paper. In particular, the extension of the $H$-space result to oddly generated elliptic spaces is an observation of the referee.

\section{REFERENCES}

[Bro] R. F. Brown, The Lefschetz Fixed Point Theorem, Scott, Foresman and Co., 1971. MR 44:1023

[G-M] P. Griffiths and J. Morgan, Rational Homotopy Theory and Differential Forms, Birkhäuser, 1981. MR 82m:55014

[Dua 1 ] Duan H., The Lefschetz Number of Self-Maps of Lie Groups, Proc. A. M. S. 104 (1988), 1284-1286. MR 89d:55002

[Dua 2$]$ Duan H., A characteristic polynomial for self-maps of $H$-spaces, Quart. J. Math. Oxford 44 (2) (1993), 315-325. MR 94j:55003

[Hal] S. Halperin, Spaces whose rational homology and de Rham homotopy are both finite dimensional, Astérisque: Homotopie algèbrique et algébra locale 113-114 (1984), Soc. Math. France, 198-205. MR 86a:55014

[Hun] T. Hungerford, Abstract Algebra, An Introduction, Saunders, 1990.

[Lan] S. Lang, Algebra, corrected reprint, Addison Wesley, 1971. MR 33:5416

[Spa] E. Spanier, Algebraic Topology, McGraw-Hill, 1966. MR 35:1007

Department of Mathematics, Cleveland State University, Cleveland, Ohio 44115

E-mail address: Lupton@math.csuohio.edu

E-mail address: Oprea@math.csuohio.edu 